\title{
Supersymmetry of the chiral de Rham complex
}

\author{
David Ben-Zvi, Reimundo Heluani and Matthew Szczesny
}

\begin{abstract}
We present a superfield formulation of the chiral de Rham complex (CDR), as introduced by Malikov, Schechtman and Vaintrob in 1999, in the setting of a general smooth manifold, and use it to endow CDR with superconformal structures of geometric origin. Given a Riemannian metric, we construct an $N=1$ structure on CDR (action of the $N=1$ superVirasoro, or Neveu-Schwarz, algebra). If the metric is Kähler, and the manifold Ricci-flat, this is augmented to an $N=2$ structure. Finally, if the manifold is hyperkähler, we obtain an $N=4$ structure. The superconformal structures are constructed directly from the Levi-Civita connection. These structures provide an analog for CDR of the extended supersymmetries of nonlinear $\sigma$-models.
\end{abstract}

\section{Introduction}

In the paper [MSV99], Malikov, Schechtman and Vaintrob introduced a sheaf of vertex superalgebras $\Omega_{M}^{\text {ch }}$ attached to any smooth complex variety $M$, called the chiral de Rham complex (CDR) of $M$ (see also [GMS04]). If $M$ is $n$-dimensional, the fibers of $\Omega_{M}^{\text {ch }}$ are isomorphic as vertex superalgebras to a completion of the $b c-\beta \gamma$ system on $n$ generators, or, in physics terminology, to the tensor product of the bosonic and fermionic ghost systems on $n$ generators. The sheaf cohomology of $\Omega_{M}^{\mathrm{ch}}$, $H^{*}\left(M, \Omega_{M}^{\text {ch }}\right)$, also a vertex superalgebra, is related to the chiral algebra of the half-twist of the $\sigma$ model with target $M$, a quantum field theory associated to $M$ (see [FL07], [Kap05] and [Wit05]). It is shown in [MSV99] that, in the holomorphic setting, for arbitrary $M, H^{*}\left(M, \Omega_{M}^{\text {ch }}\right)$ carries a conformal structure, and when $M$ has a global holomorphic volume form, $H^{*}\left(M, \Omega_{M}^{\text {ch }}\right)$ admits $N=2$ superconformal symmetry (equivalently, admits the structure of a topological vertex algebra).

In this paper, we present a superfield construction of the chiral de Rham complex in the $C^{\infty}$ setting, and examine how various geometric structures on a manifold give rise to extra symmetries on $\Omega_{M}^{\text {ch }}$. (For the $C^{\infty}$ formulation of CDR, see [MSV99] and [LL07] - we present a streamlined formulation in $\S 5$. For a related super-spacetime approach to CDR, see [GMS03].) We show that a Riemannian metric on $M$ gives rise to an $N=1$ structure on $\Omega_{M}^{\text {ch }}$, i.e. there exist global sections of $\Omega_{M}^{\text {ch }}$ that generate an $N=1$ superconformal vertex subalgebra. The $N=1$ structure is constructed from the Levi-Civita connection on $M$. When $M$ is Ricci-flat and Kähler, this is augmented to an $N=2$ structure (quasiclassical limits of the $N=1$ and $N=2$ structures on CDR were independently obtained by Malikov (private communication), starting from the Lagrangian for the $\sigma$-model). In this case, choosing holomorphic and anti-holomorphic coordinates on $M, \Omega_{M}^{\text {ch }}$ can be locally written as a tensor product of a holomorphic and anti-holomorphic part. The $N=2$ structure splits as a tensor product of two commuting $N=2$ vertex algebras, one 'holomorphic' and another

Received 25 April 2006, accepted in final form 16 July 2007, published online 7 February 2008.

2000 Mathematics Subject Classification 17B69, 81T60, 53C26.

Keywords: chiral de Rham, hyperkähler.

The first author was supported by a Summer Research Assignment from the University of Texas, and the third author was supported by NSF grant DMS-0401619.

This journal is (C) Foundation Compositio Mathematica 2008. 


\section{Ben-Zvi, R. Heluani And M. Szczesny}

'anti-holomorphic'. These structures are slightly different from the ones considered in [MSV99], and agree only in the case when the metric $g$ is flat.

Finally, we consider the case where $M$ is a hyperkähler manifold. Recall that a hyperkähler manifold is a Riemannian manifold possessing three isometric complex structures $I, J$ and $K$, parallel with respect to the Levi-Civita connection, and satisfying the quaternionic relations

$$
I^{2}=J^{2}=K^{2}=-\mathrm{Id}, \quad I J=-J I=K .
$$

The real dimension of $M$ is then necessarily a multiple of 4 , and the above is equivalent to the holonomy group of $M$ being contained in $S p(n, \mathbb{H}) \subset S O(4 n, \mathbb{R})$. We show that, in this case, the sheaf $\Omega_{M}^{\text {ch }}$ carries $N=4$ superconformal symmetry, i.e. has an embedding of the $N=4$ superconformal vertex algebra.

Our approach relies heavily on the superfield formalism for vertex algebras introduced in [HK07] under the moniker of SUSY vertex algebras. In the standard approach to vertex superalgebras one considers fields which are endomorphism-valued distributions,

$$
A(z)=\sum_{n \in \mathbb{Z}} z^{-n-1} A_{(n)} \in \text { End } V\left[\left[z, z^{-1}\right]\right], \quad A_{(n)} \in \text { End } V,
$$

where $V$ is the vertex superalgebra. Let $\theta$ be an odd formal variable satisfying $\theta^{2}=0$. A superfield is an endomorphism-valued distribution of the form

$$
A^{s}(z, \theta)=A(z)+\theta B(z), \quad A(z), B(z) \in \text { End } V\left[\left[z, z^{-1}\right]\right] .
$$

The operator products of the two fields $A(z)$ and $B(z)$ are now encoded in the operator product of the single superfield $A^{s}(z, \theta)$. In the standard field approach to vertex superalgebras, the $N=4$ superconformal algebra is generated by eight fields. Thus, constructing a representation of this object in terms of free fields involves checking that a very large number of operator products are correct. In the SUSY vertex algebra formalism, the $N=4$ superconformal algebra is generated by only four fields, which greatly simplifies the computations involved.

The SUSY vertex algebra formalism also yields a simpler, tensorial description of the chiral de Rham complex. In a coordinate patch $U$ diffeomorphic to $\mathbb{R}^{n}, \Omega_{X}^{\text {ch }}(U)$ is generated by sections $a^{i}(z), b^{i}(z), \phi^{i}(z), \psi^{i}(z), i=1, \ldots, n$. Under a change of coordinates, $b^{i}(z)$ transforms as a function, $\phi^{i}(z)$ as a 1-form and $\psi^{i}(z)$ as a vector field, while $a^{i}(z)$ transforms in a seemingly complicated, nonlinear way (see Equation (4.1.3)). In the SUSY formalism, these are combined into two superfields:

$$
B^{i}(z, \theta)=b^{i}(z)+\theta \phi^{i}(z), \quad \Psi^{i}(z, \theta)=\psi^{i}(z)+\theta a^{i}(z) .
$$

We show that, under a change of coordinates, $B^{i}(z, \theta)$ transforms as a function and $\Psi^{i}(z, \theta)$ simply as a vector field. In particular, the well-known cancellation of anomalies necessary for the construction of the chiral de Rham complex becomes an obvious consequence of the superfield formalism.

To construct the desired supersymmetries, we first show that endomorphisms of the tangent bundle give sections of the chiral de Rham complex, and hence (under the state-field correspondence) fields or superfields. The basic $N=1$ structure (Neveu-Schwarz current) of CDR on any Riemannian manifold is constructed explicitly out of the metric. Finally, to enhance this to an $N=2$ (respectively, $N=4$ ) structure on a Calabi-Yau (respectively, hyperkähler) manifold, we simply adjoin the superfield associated to the complex structure endomorphism $I$ (respectively, the superfields associated to $I, J$ and $K$ ).

The superconformal structures exhibited in this paper are inspired by the well-known supersymmetries of $\sigma$-models. Zumino [Zum79] and Alvarez-Gaumé and Freedman [AGF81] showed that nonlinear $\sigma$-models on general Riemannian, complex and hyperkähler targets carry $N=1,2,4$ super-Poincaré symmetries, respectively (see [HKLR87, Fre99] for excellent discussions, and [BGL04] for a related recent development). In the $N=2$ case, this structure is superconformal when the 


\section{Supersymmetry of the ChIRAL De Rham COMPleX}

target is Calabi-Yau. (Note that the quantum $\sigma$-model is only conformally invariant, up to higher order terms, when the Ricci curvature vanishes.)

It is interesting to compare the superconformal structures we exhibit with the bundles of superconformal vertex algebras constructed by Tamanoi [Tam99] and Zhou [Zho00]. Note that these bundles of vertex algebras are linear, i.e. associated to the (frame bundles of the) tangent bundles of Riemannian manifolds, and should be compared with the associated graded of our construction with respect to a natural filtration on CDR (which depends on all order jets on the manifold). Note also that [MSV99] endow CDR with an $N=2$ structure that is defined for any smooth manifold, coming from the choice of a volume form on that manifold. However, this structure is different from ours (and that for the $\sigma$-model), which depends directly on the metric, and does not seem to allow for an $N=4$ extension in the hyperkähler case.

Finally, the superfield formulation of the chiral de Rham complex is closely related to its geometric formulation as a factorization algebra over supercurves, extending the factorization structure over (even) curves described by Kapranov and Vasserot [KV04]. This geometric construction was an initial motivation for this work and we plan to return to it in the future.

The outline of the paper is as follows. In $\S 2$ we recall the standard approach to vertex superalgebras following [Kac96], and review the $N=1,2,4$ superconformal vertex algebras in this language. In $\S 3$ we introduce the formalism of SUSY vertex algebras following [HK07], and give several examples, including a description of the $N=1,2,4$ superconformal vertex algebras in this more compact language. Section 4 recalls the construction of the chiral de Rham complex and gives its simplified superfield description. Section 5 presents a coordinate-free construction of CDR on general smooth manifolds. Section 6 is a brief review of hyperkähler manifolds. Finally, $\S 7$ contains the construction of the $N=1,2,4$ structures on the chiral de Rham complex.

\section{Vertex superalgebras}

In this section, we review the definition of vertex superalgebras, as presented in [Kac96], in order to fix notation, and facilitate comparison with the superfield formalism introduced later.

2.1. Given a vector space $V$, an $\operatorname{End}(V)$-valued field is a formal distribution of the form

$$
A(z)=\sum_{n \in \mathbb{Z}} z^{-1-n} A_{(n)}, \quad A_{(n)} \in \operatorname{End}(V),
$$

such that, for every $v \in V$, we have $A_{(n)} v=0$ for large enough $n$.

Definition 2.2. A vertex superalgebra consists of the data of a supervector space $V$, an even vector $|0\rangle \in V$ (the vacuum vector), an even endomorphism $T$, and a parity preserving linear map $A \mapsto Y(A, z)$ from $V$ to $\operatorname{End}(V)$-valued fields (the state-field correspondence). These data should satisfy the following set of axioms.

(i) Vacuum axioms:

$$
\begin{aligned}
Y(|0\rangle, z) & =\mathrm{Id}, \\
Y(A, z)|0\rangle & =A+O(z), \\
T|0\rangle & =0 .
\end{aligned}
$$

(ii) Translation invariance:

(iii) Locality:

$$
[T, Y(A, z)]=\partial_{z} Y(A, z)
$$

$$
(z-w)^{n}[Y(A, z), Y(B, w)]=0, \quad n \gg 0 .
$$

(The notation $O(z)$ denotes a power series in $z$ without constant term.) 


\section{Ben-Zvi, R. Heluani And M. Szczesny}

2.3. Given a vertex super-algebra $V$ and a vector $A \in V$, we expand the fields

$$
Y(A, z)=\sum_{j \in \mathbb{Z}} z^{-1-j} A_{(j)}
$$

and we call the endomorphisms $A_{(j)}$ the Fourier modes of $Y(a, z)$. Define now the operations

$$
\begin{aligned}
{\left[A_{\lambda} B\right] } & =\sum_{j \geqslant 0} \frac{\lambda^{j}}{j !} A_{(j)} B, \\
A B & =A_{(-1)} B .
\end{aligned}
$$

The first operation is called the $\lambda$-bracket and the second is called the normally ordered product. The $\lambda$-bracket contains all of the information about the commutators between the Fourier coefficients of fields in $V$.

\section{The $N=1, N=2$ and $N=4$ superconformal vertex algebras}

In this section we review the standard description of the $N=1,2,4$ superconformal vertex algebras. In $\S 3$, the same algebras will be described in the SUSY vertex algebra formalism.

Example 2.4 (The $N=1$ (Neveu-Schwarz) superconformal vertex algebra). The $N=1$ superconformal vertex algebra [Kac96] of central charge $c$ is generated by two fields: $L(z)$, an even field of conformal weight 2 , and $G(z)$, an odd primary field of conformal weight $\frac{3}{2}$, with the $\lambda$-brackets

$$
\begin{gathered}
{\left[L_{\lambda} L\right]=(T+2 \lambda) L+\frac{c \lambda^{3}}{12},} \\
{\left[L_{\lambda} G\right]=\left(T+\frac{3}{2} \lambda\right) G,} \\
{\left[G_{\lambda} G\right]=2 L+\frac{c \lambda^{2}}{3} .}
\end{gathered}
$$

Here $L(z)$ is called the Virasoro field.

Example 2.5 (The $N=2$ superconformal vertex algebra). The $N=2$ superconformal vertex algebra of central charge $c$ is generated by the Virasoro field $L(z)$ with $\lambda$-bracket (2.4.1), an even primary field $J(z)$ of conformal weight 1 , and two odd primary fields $G^{ \pm}(z)$ of conformal weight $\frac{3}{2}$, with the $\lambda$-brackets $[\operatorname{Kac} 96]$

$$
\begin{gathered}
{\left[L_{\lambda} J\right]=(T+\lambda) J,} \\
{\left[L_{\lambda} G^{ \pm}\right]=\left(T+\frac{3}{2} \lambda\right) G^{ \pm},} \\
{\left[J_{\lambda} G^{ \pm}\right]= \pm G^{ \pm},} \\
{\left[G_{\lambda}^{+} G^{-}\right]=L+\frac{1}{2} T J+\lambda J+\frac{c}{6} \lambda^{2}, \quad\left[G_{\lambda}^{ \pm} G^{ \pm}\right]=0 .}
\end{gathered}
$$

Example 2.6 (The 'small' $N=4$ superconformal vertex algebra). The even part of this vertex algebra is generated by the Virasoro field $L(z)$ and three primary fields of conformal weights $1, J^{0}$, $J^{+}$and $J^{-}$. The odd part is generated by four primary fields of conformal weight $\frac{3}{2}, G^{ \pm}$and $\bar{G}^{ \pm}$. 


\section{SUPERSYMMETRY OF THE CHIRAL DE RHAM COMPLEX}

The remaining (non-vanishing) $\lambda$-brackets are (cf. [KW04, p. 36])

$$
\begin{array}{rlrl}
{\left[J_{\lambda}^{0} J^{ \pm}\right]} & = \pm 2 J^{ \pm}, & {\left[J_{\lambda}^{0} J^{0}\right]} & =\frac{c}{3} \lambda, \\
{\left[J_{\lambda}^{+} J^{-}\right]} & =J^{0}+\frac{c}{6} \lambda, & {\left[J_{\lambda}^{0} G^{ \pm}\right]} & = \pm G^{ \pm}, \\
{\left[J_{\lambda}^{0} \bar{G}^{ \pm}\right]} & = \pm \bar{G}^{ \pm}, & {\left[J_{\lambda}^{+} G^{-}\right]} & =G^{+}, \\
{\left[J_{\lambda}^{-} G^{+}\right]} & =G^{-}, & {\left[J_{\lambda}^{+} \bar{G}^{-}\right]} & =-\bar{G}^{+}, \\
{\left[J_{\lambda}^{-} \bar{G}^{+}\right]} & =-\bar{G}_{\lambda}^{ \pm}, & \left.\bar{G}^{ \pm}\right] & =(T+2 \lambda) \\
{\left[G_{\lambda}^{ \pm} \bar{G}^{\mp}\right]} & =L \pm \frac{1}{2} T J^{0} \pm \lambda J^{0}+\frac{c}{6} \lambda^{2} . &
\end{array}
$$

(Note that the $J$ currents form an $\mathfrak{s l}_{2}$ current algebra.)

\section{SUSY vertex algebras}

In this section we collect some results on SUSY vertex algebras (SUSY VAs) from [HK07]. Since we only need the case with one odd variable, we will adapt the notation to this case, and avoid the prefix 'super' when possible.

\section{Structure theory of SUSY VAs}

3.1. Let us fix notation first. We introduce formal variables $Z=(z, \theta)$ and $W=(w, \zeta)$, where $\theta, \zeta$ are odd anticommuting variables and $z, w$ are even commuting variables. Given an integer $j$ and $J=0$ or 1 we put $Z^{j \mid J}=z^{j} \theta^{J}$.

Let $\mathscr{H}$ be the universal enveloping algebra of the $1 \mid 1$ dimensional Lie super-algebra $[\chi, \chi]=-2 \lambda$, where $\chi$ is odd and $\lambda$ is even and central (super-Heisenberg or Clifford algebra). We will consider another set of generators $-S$ and $-T$ for $\mathscr{H}$, where $S$ is odd, $T$ is central, and $[S, S]=2 T$. Whenever we treat $\mathscr{H}$ as an $\mathscr{H}$-module it will be by the adjoint action. Denote $\Lambda=(\lambda, \chi), \nabla=(T, S)$, $\Lambda^{j \mid J}=\lambda^{j} \chi^{J}$ and $\nabla^{j \mid J}=T^{j} S^{J}$.

Given a supervector space $V$ and a vector $a \in V$, we will denote by $(-1)^{a}$ its parity.

3.2. Let $U$ be a vector space. A $U$-valued formal distribution is an expression of the form

$$
\sum_{\substack{j \in \mathbb{Z} \\ J=0,1}} Z^{-1-j \mid 1-J} w_{(j \mid J)}, \quad w_{(j \mid J)} \in U .
$$

The space of such distributions will be denoted by $U\left[\left[Z, Z^{-1}\right]\right]$. If $U$ is a Lie algebra, we will say that two such distributions $a(Z)$ and $b(W)$ are local if

$$
(z-w)^{n}[a(Z), b(W)]=0, \quad n \gg 0 .
$$

The space of distributions such that only finitely many negative powers of $z$ appear (i.e. $w_{(j \mid J)}=0$ for large enough $j$ ) will be denoted $U((Z))$. In the case when $U=\operatorname{End}(V)$ for another vector space $V$, we will say that a distribution $a(Z)$ is a field if $a(Z) v \in V((Z))$ for all $v \in V$.

Definition 3.3. An $N=1$ SUSY vertex algebra consists of the data of a vector space $V$, an even vector $|0\rangle \in V$ (the vacuum vector), an odd endomorphism $S$ (whose square is an even endomorphism that we denote by $T$ ), and a parity preserving linear map $A \mapsto Y(A, Z)$ from $V$ to $\operatorname{End}(V)$-valued fields (the state-field correspondence). These data should satisfy the following set of axioms. 
(i) Vacuum axioms:

$$
\begin{aligned}
Y(|0\rangle, Z) & =\mathrm{Id}, \\
Y(A, Z)|0\rangle & =A+O(Z), \\
S|0\rangle & =0 .
\end{aligned}
$$

(ii) Translation invariance:

$$
\begin{aligned}
{[S, Y(A, Z)] } & =\left(\partial_{\theta}-\theta \partial_{z}\right) Y(A, Z) \\
{[T, Y(A, Z)] } & =\partial_{z} Y(A, Z)
\end{aligned}
$$

(iii) Locality:

$$
(z-w)^{n}[Y(A, Z), Y(B, W)]=0, \quad n \gg 0 .
$$

Remark 3.4. Given the vacuum axiom for a SUSY vertex algebra, we will use the state-field correspondence to identify a vector $A \in V$ with its corresponding field $Y(A, Z)$.

3.5. Given an $N=1$ SUSY vertex algebra $V$ and a vector $A \in V$, we expand the fields

$$
Y(A, Z)=\sum_{\substack{j \in \mathbb{Z} \\ J=0,1}} Z^{-1-j \mid 1-J} A_{(j \mid J)}
$$

and we call the endomorphisms $A_{(j \mid J)}$ the Fourier modes of $Y(A, Z)$. Define now the operations

$$
\begin{aligned}
{\left[A_{\Lambda} B\right] } & =\sum_{\substack{j \geqslant 0 \\
J=0,1}} \frac{\Lambda^{j \mid J}}{j !} A_{(j \mid J)} B, \\
A B & =A_{(-1 \mid 1)} B .
\end{aligned}
$$

The first operation is called the $\Lambda$-bracket and the second is called the normally ordered product.

Remark 3.6. As in the standard setting, given a SUSY VA $V$ and a vector $A \in V$, we have

$$
Y(T A, Z)=\partial_{z} Y(A, Z)=[T, Y(A, Z)] \text {. }
$$

On the other hand, the action of the 'odd' derivation $S$ is described by

$$
Y(S A, Z)=\left(\partial_{\theta}+\theta \partial_{z}\right) Y(A, Z) \neq[S, Y(A, Z)] .
$$

The relation with the standard field formalism is as follows. Suppose that $V$ is a vertex superalgebra as defined in $\S 2$, together with a homomorphism from the $N=1$ superconformal vertex algebra in Example 2.4. Therefore $V$ possesses an even vector $\nu$ of conformal weight 2, and an odd vector $\tau$ of conformal weight $\frac{3}{2}$, whose associated fields

$$
\begin{aligned}
& Y(\nu, z)=L(z)=\sum_{n \in \mathbb{Z}} L_{n} z^{-n-2}, \\
& Y(\tau, z)=G(z)=\sum_{n \in \frac{1}{2}+\mathbb{Z}} G_{n} z^{-n-\frac{3}{2}}
\end{aligned}
$$

have the $\lambda$-brackets as in Example 2.4, and where we require $G_{-1 / 2}=S$ and $L_{-1}=T$. We can then endow $V$ with the structure of an $N=1$ SUSY vertex algebra via the state-field correspondence $[$ Kac96]

$$
Y(A, Z)=Y^{\mathrm{c}}(A, z)+\theta Y^{\mathrm{c}}\left(G_{-1 / 2} A, z\right),
$$

where we have written $Y^{\mathrm{c}}$ to emphasize that this is the 'classical' state-field (rather than statesuperfield) correspondence in the sense of $\S 2$. 


\section{Supersymmetry of the ChIRAL De Rham COMPleX}

(Note however that there exist SUSY vertex algebras without such a map from the $N=1$ superconformal vertex algebra.)

Definition 3.7. Let $\mathscr{H}$ be as before. An $N=1 S U S Y$ Lie conformal algebra is an $\mathscr{H}$-module $\mathscr{R}$ with an operation $[\Lambda]: \mathscr{R} \otimes \mathscr{R} \rightarrow \mathscr{H} \otimes \mathscr{R}$ of degree 1 satisfying the following.

(i) Sesquilinearity:

$$
\left[S a_{\Lambda} b\right]=\chi\left[a_{\Lambda} b\right], \quad\left[a_{\Lambda} S b\right]=-(-1)^{a}(S+\chi)\left[a_{\Lambda} b\right]
$$

(ii) Skew-symmetry:

$$
\left[b_{\Lambda} a\right]=(-1)^{a b}\left[b_{-\Lambda-\nabla} a\right] .
$$

Here the bracket on the right-hand side is computed as follows: first compute $\left[b_{\Gamma} a\right]$, where $\Gamma=(\gamma, \eta)$ are generators of $\mathscr{H}$ supercommuting with $\Lambda$, then replace $\Gamma$ by $(-\lambda-T,-\chi-S)$.

(iii) Jacobi identity:

$$
\left[a_{\Lambda}\left[b_{\Gamma} c\right]\right]=-(-1)^{a}\left[\left[a_{\Lambda} b\right]_{\Gamma+\Lambda} c\right]+(-1)^{(a+1)(b+1)}\left[b_{\Gamma}\left[a_{\Lambda} c\right]\right] .
$$

Here the first bracket on the right-hand side is computed as in skew-symmetry and the identity is an identity in $\mathscr{H}^{\otimes 2} \otimes \mathscr{R}$.

Morphisms of SUSY Lie conformal algebras are $\mathscr{H}$-module morphisms $\varphi: \mathscr{R} \rightarrow \mathscr{R}^{\prime}$ such that the following diagram is commutative.

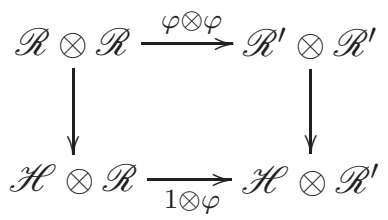

Remark 3.8. In this definition we consider $\mathscr{R} \otimes \mathscr{R}$ as a module over $\mathscr{H}$ using the co-multiplication of $\mathscr{H}$. Similarly $\mathscr{H} \otimes \mathscr{R}$ is a module over $\mathscr{H}$ (recall that $\mathscr{H}$ is a module over itself with the adjoint action). The bracket $[\Lambda]$ is a morphism of $\mathscr{H}$-modules. The Jacobi identity is an identity in $\mathscr{H} \otimes \mathscr{H} \otimes \mathscr{R}$.

3.9. Given an $N=1$ SUSY VA, it is canonically an $N=1$ SUSY Lie conformal algebra with the bracket defined in (3.5.2). Moreover, given an $N=1$ Lie conformal algebra $\mathscr{R}$, there exists a unique $N=1$ SUSY VA called the universal enveloping SUSY vertex algebra of $\mathscr{R}$ with the property that, if $W$ is another $N=1$ SUSY VA and $\varphi: \mathscr{R} \rightarrow W$ is a morphism of Lie conformal algebras, then $\varphi$ extends uniquely to a morphism $\varphi: V \rightarrow W$ of SUSY VAs.

3.10. The operations (3.5.2) satisfy the following.

(i) Quasi-commutativity:

$$
a b-(-1)^{a b} b a=\int_{-\nabla}^{0}\left[a_{\Lambda} b\right] d \Lambda .
$$

(ii) Quasi-associativity:

$$
(a b) c-a(b c)=\sum_{j \geqslant 0} a_{(-j-2 \mid 1)} b_{(j \mid 1)} c+(-1)^{a b} \sum_{j \geqslant 0} b_{(-j-2 \mid 1)} a_{(j \mid 1)} c .
$$

(iii) Quasi-Leibniz (non-commutative Wick formula):

$$
\left[a_{\Lambda} b c\right]=\left[a_{\Lambda} b\right] c+(-1)^{(a+1) b} b\left[a_{\Lambda} c\right]+\int_{0}^{\Lambda}\left[\left[a_{\Lambda} b\right]_{\Gamma} c\right] d \Gamma .
$$




\section{Ben-Zvi, R. Heluani And M. Szczesny}

Here the integral $\int d \Lambda$ is $\partial_{\chi} \int d \lambda$. In addition, the vacuum vector is a unit for the normally ordered product and the endomorphisms $S$ and $T$ are odd and even derivations respectively of both operations.

\section{Examples}

Example 3.11. Let $\mathscr{R}$ be the free $\mathscr{H}$-module generated by an odd vector $H$. Consider the following Lie conformal algebra structure in $\mathscr{R}$ :

$$
\left[H_{\Lambda} H\right]=(2 T+\chi S+3 \lambda) H .
$$

This is the Neveu-Schwarz algebra (of central charge 0). This algebra admits a central extension of the form

$$
\left[H_{\Lambda} H\right]=(2 T+\chi S+3 \lambda) H+\frac{c}{3} \chi \lambda^{2},
$$

where $c$ is any complex number. The associated universal enveloping SUSY VA is the Neveu-Schwarz algebra of central charge $c .{ }^{1}$ If we decompose the corresponding field

$$
H(z, \theta)=G(z)+2 \theta L(z),
$$

then the fields $G(z)$ and $L(z)$ satisfy the commutation relations of the well-known $N=1$ supervertex algebra in Example 2.4.

Example 3.12. Consider now the free $\mathscr{H}$-module generated by even vectors $\left\{B^{i}\right\}_{i=1}^{n}$ and odd vectors $\left\{\Psi^{i}\right\}_{i=1}^{n}$, where the only non-trivial commutation relations are

$$
\left[B_{\Lambda}^{i} \Psi^{j}\right]=\delta_{i j}=\left[\Psi_{\Lambda}^{j} B^{i}\right] .
$$

Expand the corresponding fields as

$$
B^{i}(z, \theta)=b^{i}(z)+\theta \phi^{i}(z), \quad \Psi^{i}(z, \theta)=\psi^{i}(z)+\theta a^{i}(z) .
$$

Then the fields $b^{i}, a^{i}, \phi^{i}$ and $\psi^{i}$ generate the $b c-\beta \gamma$ system as in [MSV99].

Example 3.13. The $N=2$ superconformal vertex algebra is generated by four fields [Kac96]. In this context it is generated by two superfields - an $N=1$ vector $H$ as in Example 3.11 and an even current $J$, primary of conformal weight 1, i.e.

$$
\left[H_{\Lambda} J\right]=(2 T+2 \lambda+\chi S) J .
$$

The remaining commutation relation is

$$
\left[J_{\Lambda} J\right]=-\left(H+\frac{c}{3} \lambda \chi\right) .
$$

Note that given the current $J$ we can recover the $N=1$ vector $H$. In terms of the fields of Example 2.5, $H$ and $J$ decompose as

$$
\begin{aligned}
J(z, \theta) & =-\sqrt{-1} J(z)-\sqrt{-1} \theta\left(G^{-}(z)-G^{+}(z)\right), \\
H(z, \theta) & =\left(G^{+}(z)+G^{-}(z)\right)+2 \theta L(z) .
\end{aligned}
$$

Example 3.14. The 'small' $N=4$ superconformal vertex algebra is a vertex algebra generated by eight fields [Kac96]. In this formalism, it is generated by four superfields $H, J^{i}, i=0,1,2$, such that each pair $\left(H, J^{i}\right)$ forms an $N=2$ SUSY VA as in the previous example and the remaining commutation relations are

$$
\left[J_{\Lambda}^{i} J^{j}\right]=\varepsilon^{i j k}(S+2 \chi) J^{k}, \quad i \neq j,
$$

\footnotetext{
${ }^{1}$ Properly speaking, we consider the universal enveloping SUSY vertex algebra of $\mathscr{R} \oplus \mathbb{C} C$ with $C$ central and $T C=$ $S C=0$ and then we quotient by the ideal generated by $C=c$ for any complex number $c$.
} 


\section{Supersymmetry OF THE CHIRAL DE RHAM COMPLEX}

where $\varepsilon$ is the totally antisymmetric tensor. (In other words, we are writing the $N=4$ algebra in terms of an $\mathfrak{s} u_{2}$ basis $J^{i}$ of superfields rather than the $\mathfrak{s l}_{2}$ basis $J^{0}, J^{ \pm}$of even fields, together with odd $G$ fields, as before.) In terms of the fields of Example 2.6, $H$ and $J^{i}$ decompose as

$$
\begin{aligned}
& J^{0}(z, \theta)=-\sqrt{-1} J^{0}(z)-\sqrt{-1} \theta\left(\bar{G}^{-}(z)-G^{+}(z)\right), \\
& J^{1}(z, \theta)=\sqrt{-1}\left(J^{+}(z)+J^{-}(z)\right)+\sqrt{-1}\left(\bar{G}^{+}(z)-G^{-}(z)\right), \\
& J^{2}(z, \theta)=\left(J^{+}(z)-J^{-}(z)\right)+\theta\left(\bar{G}^{+}(z)+G^{-}(z)\right), \\
& H(z, \theta)=\left(G^{+}(z)+\bar{G}^{-}(z)\right)+2 \theta L(z) .
\end{aligned}
$$

\section{Chiral de Rham complex}

In this section we recollect some results from [MSV99]. We then provide and discuss a superfield formulation of the chiral de Rham complex in the algebraic setting. For the applications we have in mind, we will need to work in the $C^{\infty}$ setting, which is described in $\S 5$.

4.1. The chiral de Rham complex $\Omega_{M}^{\mathrm{ch}}$ is a sheaf of vertex algebras defined over any smooth algebraic variety $M$ over the complex numbers. In order to construct such a sheaf, the authors in [MSV99] first construct a sheaf of supervertex algebras on $\mathbb{C}^{n}$ and then show that we can glue these sheaves by studying the action of changes of coordinates.

To construct the sheaf on $U=\operatorname{Spec} \mathbb{C}\left[x^{1}, \ldots, x^{n}\right]$, we first look at its global sections. This vertex algebra can be described in terms of generators and relations as follows. Then $\Omega^{\text {ch }}(U)$ is the $b c-\beta \gamma$ system vertex algebra. Namely, it is generated by fields $\left\{a^{i}, b^{i}, \psi^{i}, \phi^{i}\right\}_{i=1}^{n}$, with commutation relations

$$
\left[a_{\lambda}^{i} b^{j}\right]=\left[\phi_{\lambda}^{i} \psi^{j}\right]=\delta_{i j},
$$

where we have identified the coordinate functions $x^{i}$ with the $(-1)$ Fourier mode of the fields $b^{i}(z)$ (recall that we identify vectors in our vertex algebras with the corresponding fields by the state-field correspondence).

The next step in [MSV99] is to consider a localization of this vertex algebra, whereby we allow expressions of the form

$$
f\left(b^{1}(z), \ldots, b^{n}(z)\right)
$$

where $f\left(x^{1}, \ldots, x^{n}\right)$ is an arbitrary algebraic function on $U$. This allows us to construct a Zariski sheaf on $\mathbb{C}^{n}$. We may also pass to a formal completion, allowing $f$ to be an arbitrary function on the formal disk $\operatorname{Spf} \mathbb{C}\left[\left[x^{1}, \ldots, x^{n}\right]\right]$.

Finally, in order to glue these sheaves, one has to analyze how these generators transform under changes of coordinates of the formal disk. Given such a change of coordinates $\tilde{x}^{i}=g^{i}(x)$, with inverse $x^{i}=f^{i}(\tilde{x})$, the generating fields transform as

$$
\begin{aligned}
\tilde{b}^{i} & =g^{i}(b), \\
\tilde{\phi}^{i} & =\left(\frac{\partial g^{i}(b)}{\partial b^{j}} \phi^{j}\right), \\
\tilde{\psi}^{i} & =\left(\frac{\partial f^{j}}{\partial \tilde{b}^{i}}(g(b)) \psi^{j}\right), \\
\tilde{a}^{i} & =\left(a^{j} \frac{\partial f^{j}}{\partial \tilde{b}^{i}}(g(b))\right)+\left(\frac{\partial^{2} f^{k}}{\partial \tilde{b}^{j} \partial \tilde{b}^{l}}(g(b)) \frac{\partial g^{l}}{\partial b^{r}} \phi^{r} \psi^{k}\right) .
\end{aligned}
$$

4.2. Let us analyze this sheaf of vertex algebras as a sheaf of $N=1$ SUSY VAs. For this, we combine the generators into superfields as

$$
B^{i}=b^{i}+\theta \phi^{i}, \quad \Psi^{i}=\psi^{i}+\theta a^{i} .
$$




\section{Ben-Zvi, R. Heluani And M. Szczesny}

These fields generate a SUSY VA as in Example 3.12. Given a change of coordinates $g$ as above, the formulas (4.1.3) imply that these fields transform as

$$
\begin{aligned}
\tilde{B}^{i} & =g^{i}(B), \\
\tilde{\Psi}^{i} & =\left(\frac{\partial f^{j}}{\partial \tilde{B}^{i}}(g(B)) \Psi^{j}\right) .
\end{aligned}
$$

Therefore the chiral de Rham complex is described in a simple fashion when viewed as a sheaf of SUSY VAs.

Conversely, the transformation properties (4.2.2) imply (4.1.3). Indeed, suppose that the fields $\Psi^{i}$ and $B^{i}$ transform as in (4.2.2). Evaluating at $\theta=0$ we immediately obtain the transformation properties of $\psi^{i}$ and $b^{i}$ as in (4.1.3). We now note that

$$
S \tilde{B}^{i}=S g^{i}(B)=\frac{\partial g^{i}(B)}{\partial B^{j}} S B^{j}
$$

and evaluating at $\theta=0$ we obtain the transformation property of $\phi^{i}$. Finally, we have

$$
\begin{aligned}
S \tilde{\Psi}^{i} & =S\left(\frac{\partial f^{j}}{\partial \tilde{B}^{i}}(g(B)) \Psi^{j}\right) \\
& =\left(\frac{\partial^{2} f^{j}}{\partial \tilde{B}^{i} \partial \tilde{B}^{k}}(g(B)) S \tilde{B}^{k}\right) \Psi^{j}+\frac{\partial f^{j}}{\partial \tilde{B}^{i}}(g(B)) S \Psi^{j} \\
& =\left(\frac{\partial^{2} f^{j}}{\partial \tilde{B}^{i} \partial \tilde{B}^{k}}(g(B)) \frac{\partial g^{k}(B)}{\partial B^{l}} S B^{l}\right) \Psi^{j}+\frac{\partial f^{j}}{\partial \tilde{B}^{i}}(g(B)) S \Psi^{j} .
\end{aligned}
$$

Using quasi-commutativity we can write the second term as

$$
S \Psi^{j} \frac{\partial f^{j}}{\partial \tilde{B}^{i}}(g(B))-T\left(\frac{\partial^{2} f^{j}}{\partial \tilde{B}^{i} \partial \tilde{B}^{j}}(g(B)) \frac{\partial \tilde{B}^{l}}{\partial B^{j}}\right) .
$$

On the other hand, using quasi-associativity we can write the first term of (4.2.4) as

$$
\frac{\partial^{2} f^{j}}{\partial \tilde{B}^{i} \partial \tilde{B}^{k}}(g(B)) \frac{\partial g^{k}(B)}{\partial B^{l}} S B^{l} \Psi^{j}+T\left(\frac{\partial^{2} f^{j}}{\partial \tilde{B}^{i} \partial \tilde{B}^{k}}(g(B)) \frac{\partial g^{k}(B)}{\partial B^{j}}\right) .
$$

Adding these two terms and evaluating at $\theta=0$ we obtain the transformation property of $a^{i}$.

4.2.1 From this perspective, we can construct the chiral de Rham complex as a sheaf of SUSY vertex algebras, by arguing as in [MSV99], replacing vertex algebras by SUSY VAs and using (4.2.2) instead of (4.1.3). To do this we must check that (4.2.2) preserves the SUSY VA structure. This can be done as follows. We check immediately that

$$
\left[\tilde{B}_{\Lambda}^{i} \tilde{B}^{j}\right]=0
$$

On the other hand, we have from the Wick formula:

$$
\left[\tilde{B}_{\Lambda}^{i} \tilde{\Psi}^{j}\right]=\frac{\partial f^{k}}{\partial \tilde{B}^{j}}(g(B)) \frac{\partial g^{i}(B)}{\partial\left(B^{k}\right)}=\delta_{i j}
$$

To compute $\left[\tilde{\Psi}_{\Lambda}^{i} \tilde{\Psi}^{j}\right]$ we first need

$$
\left[\tilde{\Psi}_{\Lambda}^{i} \frac{\partial f^{k}}{\tilde{B}^{j}}(g(B))\right]=\frac{\partial f^{l}}{\partial \tilde{B}^{i}}(g(B)) \frac{\partial}{\partial B^{l}}\left(\frac{\partial f^{k}}{\partial \tilde{B}^{j}}(g(B))\right)
$$

and

$$
\left[\tilde{\Psi}_{\Lambda}^{i} \Psi^{k}\right]=-\frac{\partial}{\partial B^{k}}\left(\frac{\partial f^{l}}{\partial \tilde{B}^{i}}(g(B))\right) \Psi^{l}
$$




\section{SUPERSYMMETRY OF THE CHIRAL DE RHAM COMPLEX}

Now using the Wick formula and noting that the integral term vanishes we obtain

$$
\begin{aligned}
{\left[\tilde{\Psi}_{\Lambda}^{i} \tilde{\Psi}^{j}\right] } & =\frac{\partial f^{l}}{\partial \tilde{B}^{i}}(g(B)) \frac{\partial}{\partial B^{l}}\left(\frac{\partial f^{k}}{\partial \tilde{B}^{j}}(g(B))\right) \Psi^{k}-i \leftrightarrow j \\
& =\frac{\partial f^{l}}{\partial \tilde{B}^{i}}(g(B)) \frac{\partial^{2} f^{k}}{\partial \tilde{B}^{j} \partial \tilde{B}^{m}}(g(B)) \frac{\partial g^{m}(B)}{\partial B^{l}} \Psi^{k}-i \leftrightarrow j \\
& =\frac{\partial^{2} f^{k}}{\partial \tilde{B}^{j} \partial \tilde{B}^{i}}(g(B)) \Psi^{k}-i \leftrightarrow j \\
& =0 .
\end{aligned}
$$

The equivalence of (4.1.3) and (4.2.2) shows that the sheaf constructed is in fact the chiral de Rham complex of $M$.

Remark 4.2.2. We note that in this approach all the cancellation of anomalies is contained in the fact that the integral term in the $\Lambda$-bracket (4.2.11) vanishes, which in turn is an obvious consequence of our formalism.

\section{Formal setting}

4.3. In the formal setting, the chiral de Rham complex is constructed by using the standard arguments of 'formal geometry' [MSV99, 3.9] (see also [FBZ01, ch. 17]), i.e. using an action of the Lie algebra of vector fields on the formal $n$-dimensional disk on the $b c-\beta \gamma$ system. Indeed the vector field $f\left(x_{i}\right) \partial_{x_{j}}$ acts as the residue of the field

$$
f\left(b^{i}\right) a^{j}+\sum_{k=1}^{n}\left(\partial_{x_{k}} f\right)\left(b^{i}\right) \phi^{k} \psi^{j} .
$$

In the context of SUSY vertex algebras, the vector field $f\left(x_{i}\right) \partial_{x_{j}}$ simply acts as the super-residue of the superfield

$$
f\left(B^{i}\right) \Psi_{j}
$$

Here the super-residue is defined to be

$$
\operatorname{sres}_{z, \theta} f(z, \theta)=\partial_{\theta} \operatorname{res}_{z} f(z, \theta) .
$$

\section{The $C^{\infty}$ case}

5.1. In this section we give a coordinate independent description of the chiral de Rham complex of a smooth differentiable manifold $M$. This construction is essentially a superfield reformulation of the corresponding construction in [LL07].

5.2. Let us fix notation first. Let $U$ be a differentiable manifold. Let $\mathscr{T}$ be the tangent bundle of $U$ and $\mathscr{T}^{*}$ be its cotangent bundle. We let $T=\Gamma(U, \mathscr{T})$ be the space of vector fields on $U$ and $A=\Gamma\left(U, \mathscr{T}^{*}\right)$ be the space of differentiable 1 -forms on $U$. We let $\mathscr{C}=\mathscr{C}^{\infty}(U)$ be the space of differentiable functions on $U$. Denote by

$$
\langle,\rangle: A \otimes T \rightarrow \mathscr{C}
$$

the natural pairing. Finally, we denote by $\Pi$ the functor of change of parity.

Consider now a SUSY Lie conformal algebra $\mathscr{R}$ generated by the supervector space

$$
\mathscr{C} \oplus \Pi T \oplus A \oplus \Pi A .
$$




\section{Ben-Zvi, R. Heluani And M. Szczesny}

That is, we consider differentiable functions (to be denoted $f, g, \ldots$ ) as even elements, vector fields $X, Y, \ldots$ are odd elements, and finally we have two copies of the space of differential forms. For differential forms $\alpha, \beta, \ldots \in A$ we will denote the corresponding elements of $\Pi A$ by $\bar{\alpha}, \bar{\beta}, \ldots$ The non-vanishing commutation relations in $\mathscr{R}$ are given by (up to skew-symmetry)

$$
\begin{aligned}
{\left[X_{\Lambda} f\right] } & =X(f), \\
{\left[X_{\Lambda} Y\right] } & =[X, Y]_{\mathrm{Lie}}, \\
{\left[X_{\Lambda} \alpha\right] } & =\operatorname{Lie}_{X} \alpha+\lambda\langle\alpha, X\rangle, \\
{\left[X_{\Lambda} \bar{\alpha}\right] } & =\overline{\operatorname{Lie}_{X} \alpha}+\chi\langle\alpha, X\rangle,
\end{aligned}
$$

where $[,]_{\text {Lie }}$ is the Lie bracket of vector fields and $\operatorname{Lie}_{X}$ is the action of $X$ on the space of differential forms by the Lie derivative. The fact that (5.2.3) is compatible with the Jacobi identity is a (long but) straightforward computation.

We let $R(U)$ be the corresponding universal enveloping SUSY vertex algebra of $\mathscr{R}$. As noted in [LL07], this algebra is too big. We want to impose some relations in $R(U)$. We let $1_{U}$ denote the constant function 1 in $U$. Let $d: \mathscr{C} \rightarrow A$ be the de Rham differential. Define $I(U) \subset R(U)$ to be the ideal generated by elements of the form

$$
\begin{aligned}
& f_{(-1 \mid 1)} g-(f g), \quad f_{(-1 \mid 1)} X-(f X), \quad f_{(-1 \mid 1)} \alpha-(f \alpha), \quad f_{(-1 \mid 1)} \bar{\alpha}-(\overline{f \alpha}), \\
& 1_{U}-|0\rangle, \quad T f-d f, \quad S f-\overline{d f} .
\end{aligned}
$$

Finally we define the SUSY vertex algebra as

$$
\Omega^{\mathrm{ch}}(U):=R(U) / I(U) .
$$

Expanding the superfields in terms of ordinary fields, it is straightforward to check that this supervertex algebra is just $\mathcal{Q}(U)$ in the notation of [LL07]. We therefore arrive at the following result.

THEOREM 5.3 [LL07].

(i) Let $M \subset \mathbb{R}^{n}$ be an open submanifold. The assignment $U \mapsto \Omega^{\text {ch }}(U)$ defines a (weak) sheaf of SUSY vertex algebras $\Omega_{M}^{\text {ch }}$ on $M$.

(ii) For any diffeomorphism of open sets $M^{\prime} \stackrel{\varphi}{\longrightarrow} M$ we obtain a canonical isomorphism of SUSY vertex algebras $\Omega^{\mathrm{ch}}(M) \stackrel{\Omega^{\mathrm{ch}}(\varphi)}{\longrightarrow} \Omega^{\mathrm{ch}}\left(M^{\prime}\right)$. Moreover, given diffeomorphisms $M^{\prime} \stackrel{\varphi^{\prime}}{\longrightarrow} M^{\prime} \stackrel{\varphi}{\longrightarrow} M$, we have $\Omega^{\mathrm{ch}}\left(\varphi \circ \varphi^{\prime}\right)=\Omega^{\mathrm{ch}}\left(\varphi^{\prime}\right) \circ \Omega^{\mathrm{ch}}(\varphi)$.

5.4. This theorem allows us to construct a sheaf of SUSY vertex algebras in the Grothendieck topology on $\mathbb{R}^{n}$ (generated by open embeddings). This in turn lets us attach to any smooth manifold $M$, a sheaf of SUSY vertex algebras $\Omega_{M}^{\mathrm{ch}}$. We call this sheaf the chiral de Rham complex of $M$.

Remark 5.5. In the algebraic case, this construction gives the chiral de Rham complex as described in the previous section in terms of coordinates. Indeed, we see that identifying $B^{i}$ with the field corresponding to the coordinate $x^{i}$ and $\Psi_{i}$ with the field corresponding to the vector field $\partial_{x^{i}}$, the relations defining $I(U)$ are obviously satisfied.

\section{Recollections on hyperkähler manifolds}

In this section, we briefly review the notion of a hyperkähler manifold following [Joy00]. Let $(M, g)$ be a Riemannian manifold of real dimension $2 n$, and $J$ a complex structure on $M$. The metric $g$ is Hermitian if $J$ is an isometry of $g$, i.e. if

$$
g(J u, J v)=g(u, v) \text { for tangent vectors } u, v .
$$




\section{Supersymmetry of the ChIRAL De Rham COMPleX}

Given $(M, g, J)$ we can define a 2 -form $\omega$ by

$$
\omega(v, w)=g(J v, w),
$$

and $\omega$ is called the Hermitian form of $g$. The metric $g$ is said to be Kähler if $d \omega=0$, in which case $\omega$ is called the Kähler form. The following theorem [Joy00] gives other useful characterizations of Kähler metrics.

Theorem 6.1. Let $(M, g, J)$ be as above, and $\nabla$ denote the Levi-Civita connection of $g$. Then the following conditions are equivalent:

(i) $g$ is Kähler;

(ii) $\nabla J=0$;

(iii) $\nabla \omega=0$;

(iv) the holonomy group of $g$ is contained in $U(n) \subset S O(2 n, \mathbb{R})$.

A Riemannian manifold $(M, g)$ of dimension $4 n$ is hyperkähler if it possesses three complex structures $I, J$ and $K$ such that $(M, g, I),(M, g, J)$, and $(M, g, K)$ are each Kähler, and satisfy the quaternionic relations

$$
I J=-J I=K .
$$

This is equivalent to the holonomy group of $g$ lying inside of $S p(n, \mathbb{H}) \subset S O(4 n, \mathbb{R})$. Dualizing using the metric yields three Kähler forms $\omega_{I}, \omega_{J}$ and $\omega_{K}$.

\section{Superconformal structures from geometry}

7.1. Let now $(M, g)$ be a Riemannian manifold. Denote by $\Gamma_{j_{k}}^{i}$ the Christoffel symbols of the LeviCivita connection of $M$. Let $I$ be an endomorphism of the tangent bundle of $M$, namely $I$ is a tensor of type 1,1 on $M$. Let $\omega_{i}^{j}$ be the coordinate components of such a tensor. We will systematically raise and lower indices using the metric $g$ and sum over repeated indices.

In the rest of this paper, we will adopt the following notational convention, aimed at reducing clutter.

Convention. In the $\beta \gamma-b c$ SUSY vertex algebra, expressions such as

$$
\left(\omega_{i}^{j} S B^{i}\right) \Psi_{j}+\Gamma_{j k}^{i} \omega_{i}^{j} T B^{k}
$$

correspond to the field

$$
\begin{aligned}
& \left(\omega_{i}^{j}\left(B^{1}(z, \theta), \ldots, B^{n}(z, \theta)\right)\left(S B^{i}\right)(z, \theta)\right) \Psi_{j}(z, \theta) \\
& \quad+\Gamma_{j k}^{i}\left(B^{1}(z, \theta), \ldots, B^{n}(z, \theta)\right) \omega_{i}^{j}\left(B^{1}(z, \theta), \ldots, B^{n}(z, \theta)\right)\left(T B^{k}\right)(z, \theta) .
\end{aligned}
$$

In other words, all coefficients of tensors, Christoffel symbols, etc., are being evaluated on the superfields $B^{i}(z, \theta)$. This in turn should be interpreted as follows. If $f\left(x^{1}, \ldots, x^{n}\right)$ is a $C^{\infty}$ function on an open set $U$, then

$$
\begin{aligned}
f\left(B^{1}(z, \theta), \ldots, B^{n}(z, \theta)\right) & =f\left(b^{1}(z)+\theta \phi^{1}(z), \ldots, b^{n}(z)+\theta \phi^{n}(z)\right) \\
& =f\left(b^{1}(z), \ldots, b^{n}(z)\right)+\theta \sum_{i=1}^{n} \frac{\partial f}{\partial x^{i}}\left(b^{1}(z), \ldots, b^{n}(z)\right) \phi^{i}(z) .
\end{aligned}
$$

The meaning of expressions such as $f\left(b^{1}(z), \ldots, b^{n}(z)\right)$ is explained in [MSV99] (see also $\S 5$ and [LL07]). 
LEMma 7.2. The assignment

$$
I \mapsto J=\left(\omega_{i}^{j} S B^{i}\right) \Psi_{j}+\Gamma_{j k}^{i} \omega_{i}^{j} T B^{k}
$$

defines a linear morphism

$$
\Gamma\left(M, \mathscr{E} n d\left(T_{M}\right)\right) \rightarrow \Gamma\left(M, \Omega_{M}^{\mathrm{ch}}\right) .
$$

7.3. In the case where $I$ is a complex structure on $M$ with associated Kähler form $\omega$, we will denote the corresponding current $J$ by $J_{\omega}$.

Proof of Lemma 7.2. Given that the fields $B^{i}$ transform as coordinates do, we will simplify the notation and denote

$$
\frac{\partial \tilde{B}^{i}}{\partial B^{j}}=\frac{\partial g^{i}(B)}{\partial B^{j}} .
$$

The first term in $J_{\omega}$ expressed in the coordinates $\tilde{B}^{i}$ is given by

$$
\left(\frac{\partial B^{k}}{\partial \tilde{B}^{i}} \frac{\partial \tilde{B}^{j}}{\partial B^{l}} \frac{\partial \tilde{B}^{i}}{\partial B^{m}} \omega_{k}^{l} S B^{m}\right)\left(\frac{\partial B^{n}}{\partial \tilde{B}^{j}} \Psi^{n}\right)=\left(\frac{\partial \tilde{B}^{j}}{\partial B^{l}} \omega_{k}^{l} S B^{k}\right)\left(\frac{\partial B^{n}}{\partial \tilde{B}^{j}} \Psi^{n}\right) .
$$

Using quasi-associativity $(3.10 .2)$ we see that this is

$$
\left(\omega_{i}^{j} S B^{i}\right) \Psi_{j}-\frac{\partial^{2} B^{l}}{\partial \tilde{B}^{j} \partial \tilde{B}^{m}} \frac{\partial \tilde{B}^{j}}{\partial B^{k}} \frac{\partial \tilde{B}^{m}}{\partial B^{n}} \omega_{l}^{k} T B^{n} .
$$

On the other hand, in the second term in $J_{\omega}$, there are no quasi-associativity issues and the anomalous term comes from the transformation properties of the Christoffel symbols. Indeed, the second term in $J_{\omega}$ transforms as

$$
\frac{\partial^{2} B^{l}}{\partial \tilde{B}^{j} \partial \tilde{B}^{k}} \frac{\partial \tilde{B}^{i}}{\partial B^{l}} \frac{\partial B^{m}}{\partial \tilde{B}^{i}} \frac{\partial \tilde{B}^{j}}{\partial B_{n}} \frac{\partial \tilde{B}^{k}}{\partial B^{p}} \omega_{m}^{n} T B^{p}+\Gamma_{j k}^{i} \omega_{i}^{j} T B^{k} .
$$

Adding (7.3.4) and (7.3.3) we obtain the result.

THEOREM 7.4.

(i) Let $(M, g)$ be a Riemannian manifold of dimension $n$, and $\mathbf{g}=\log \sqrt{\operatorname{det} g_{i j}}$, where $\operatorname{det} g_{i j}$ is the determinant of the metric. Then

$$
H=S B^{i} S \Psi_{i}+T B^{i} \Psi_{i}-T S \mathbf{g}
$$

generates an $N=1$ superconformal structure of central charge $3 n$. We shall refer to $H$ as the Neveu-Schwarz vector.

(ii) Let $(M, g)$ be a Calabi-Yau $2 n$-manifold with Kähler form $\omega$. Then $J_{\omega}$ and $H$ generate an $N=2$ vertex algebra structure of central charge $6 n$.

(iii) If moreover $M$ is hyperkähler of dimension $4 n$, with three Kähler structures $\omega, \eta$ and $\gamma$ such that the corresponding complex structures satisfy the quaternionic relations, then $J_{\omega}, J_{\eta}, J_{\gamma}$ and $H$ generate an $N=4$ vertex algebra of central charge $12 n$.

Proof. (i) If we write $H=H^{0}-T S \mathbf{g}$, then the fact that $H^{0}$ defines a global section of $\Omega_{M}^{\text {ch }}$ and that $H^{0}$ is a Neveu-Schwarz vector of central charge $3 n$ follows from the analogous results in [MSV99]. The fact that $T S \mathbf{g}$ is a well-defined global section follows since $M$ is orientable. To check that $H$ is indeed a Neveu-Schwarz vector (3.11.2) we compute

$$
\left[H_{\Lambda}^{0} \mathbf{g}\right]=(2 T+\chi S) \mathbf{g}
$$




\section{Supersymmetry of the ChIRAL De Rham COMPleX}

which in turn follows from the fact that $H^{0}$ is a Neveu-Schwarz vector and any function $f=f\left(B^{i}\right)$ is primary of conformal weight 0 (cf. $[\mathrm{HK} 07, \S 5.6]$ ). Using sesquilinearity we obtain

$$
\begin{aligned}
{\left[H_{\Lambda}^{0} T S \mathbf{g}\right] } & =(\lambda+T)(S+\chi)(2 T+\chi S) \mathbf{g} \\
& =(2 T+3 \lambda+\chi S) T S \mathbf{g}+\lambda^{2} S \mathbf{g}+\lambda \chi T \mathbf{g} \\
{\left[T S \mathbf{g}_{\Lambda} H^{0}\right] } & =-\lambda \chi(2 T-(\chi+S) S) \mathbf{g} \\
& =-\lambda \chi T \mathbf{g}-\lambda^{2} S \mathbf{g} .
\end{aligned}
$$

It follows then that

$$
\begin{aligned}
{\left[H_{\Lambda} H\right] } & =\left[H_{\Lambda}^{0} H^{0}\right]-\left[T S \mathbf{g}_{\Lambda} H^{0}\right]-\left[H_{\Lambda}^{0} T S \mathbf{g}\right] \\
& =(2 T+3 \lambda+\chi S) H^{0}+n \lambda^{2} \chi-(2 T+3 \lambda+\chi S) T S \mathbf{g} \\
& =(2 T+3 \lambda+\chi S) H+n \lambda^{2} \chi .
\end{aligned}
$$

(ii) To check the remaining commutation relations of the $N=2$ vertex algebra as in Example 3.13 , it is enough to do it in any coordinate system. In particular, we may choose holomorphic coordinates $\left\{x_{\alpha}\right\}$ (respectively anti-holomorphic coordinates $\left\{x_{\bar{\alpha}}\right\}$ ) for the complex structure associated to $\omega$ such that

$$
\omega_{i}^{j}=\left(\begin{array}{cc}
i \mathrm{Id} & 0 \\
0 & -i \mathrm{Id}
\end{array}\right)
$$

In this case $J=J_{\omega}$ is given by

$$
J=i S B^{\alpha} \Psi_{\alpha}-i S B^{\bar{\alpha}} \Psi_{\bar{\alpha}}+i \mathbf{g}_{, \alpha} T B^{\alpha}-i \mathbf{g}_{, \bar{\alpha}} T B^{\bar{\alpha}} .
$$

Here we have used the fact that for a Kähler manifold

$$
\Gamma_{\gamma \alpha}^{\alpha}=\mathbf{g}_{, \gamma}=\partial_{\gamma} \mathbf{g}, \quad \Gamma_{\bar{\gamma} \bar{\alpha}}^{\bar{\alpha}}=\mathbf{g}_{, \bar{\gamma}}=\partial_{\bar{\gamma}} \mathbf{g} .
$$

Let us first compute $\left[H_{\Lambda} J\right]$. For this we need

$$
\begin{aligned}
{\left[H_{\Lambda} \Psi_{\alpha}\right] } & =(2 T+\lambda+\chi S) \Psi_{\alpha}+\lambda \chi \mathbf{g}_{, \alpha}, \\
{\left[H_{\Lambda} \Psi_{\bar{\alpha}}\right] } & =(2 T+\lambda+\chi S) \Psi_{\bar{\alpha}}+\lambda \chi \mathbf{g}_{, \bar{\alpha}}, \\
{\left[H_{\Lambda} B^{\alpha}\right] } & =(2 T+\chi S) B^{\alpha} \\
{\left[H_{\Lambda} B^{\bar{\alpha}}\right] } & =(2 T+\chi S) B^{\bar{\alpha}} \\
{\left[H_{\Lambda} S B^{\alpha}\right] } & =(2 T+\lambda+\chi S) S B^{\alpha}, \\
{\left[H_{\Lambda} S B^{\bar{\alpha}}\right] } & =(2 T+\lambda+\chi S) S B^{\bar{\alpha}},
\end{aligned}
$$

which follow from the fact that $\Psi_{i}$ and $B^{i}$ are primary with respect to $H^{0}$. Using now the noncommutative Wick formula we obtain

$$
\begin{aligned}
{\left[H_{\Lambda} S B^{\alpha} \Psi_{\alpha}\right]=} & \left((2 T+\lambda+\chi S) S B^{\alpha}\right) \Psi_{\alpha}+S B^{\alpha}(2 T+\lambda+\chi S) \Psi_{\alpha} \\
& +S B^{\alpha} \lambda \chi \mathbf{g}_{, \alpha}+\int_{0}^{\Lambda}\left[(2 T+\lambda+\chi S) S B_{\Gamma}^{\alpha} \Psi_{\alpha}\right] d \Gamma \\
= & (2 T+2 \lambda+\chi S) S B^{\alpha} \Psi_{\alpha}-\lambda \chi \mathbf{g}_{, \alpha} S B^{\alpha}+\int_{0}^{\Lambda}(-2 \gamma+\lambda-\chi \eta) \eta\left[B_{\Lambda}^{\alpha} \Psi_{\alpha}\right] d \Gamma .
\end{aligned}
$$

Since the integral clearly vanishes, we obtain

$$
\left[H_{\Lambda} S B^{\alpha} \Psi_{\alpha}\right]=(2 T+2 \lambda+\chi S) S B^{\alpha} \Psi_{\alpha}-\lambda \chi \mathbf{g}_{, \alpha} S B^{\alpha} .
$$


Similarly, we compute now

$$
\begin{aligned}
{\left[\mathbf{g}_{, \alpha \Lambda} H\right] } & =S B^{i}(\chi+S) \mathbf{g}_{, \alpha i}+T B^{i} \mathbf{g}_{, \alpha i} \\
& =-\chi S \mathbf{g}_{, \alpha}+S B^{i} S \mathbf{g}_{, \alpha i}+T \mathbf{g}_{, \alpha} \\
& =(T-\chi S) \mathbf{g}_{, \alpha}-\mathbf{g}_{, \alpha i j} S B^{j} S B^{i} \\
& =(T-\chi S) \mathbf{g}_{, \alpha}, \\
{\left[H_{\Lambda} \mathbf{g}_{, \alpha}\right] } & =(2 T+\chi S) \mathbf{g}_{, \alpha} .
\end{aligned}
$$

We also have

$$
\left[H_{\Lambda} T B^{\alpha}\right]=(\lambda+T)(2 T+\chi S) B^{\alpha}=(2 T+2 \lambda+\chi S) T B^{\alpha}+\lambda \chi S B^{\alpha} .
$$

Hence using the Wick formula again and noting that the integral term trivially vanishes, we obtain

$$
\begin{aligned}
{\left[H_{\Lambda} \mathbf{g}_{, \alpha} T B^{\alpha}\right]=} & \left((2 T+\chi S) \mathbf{g}_{, \alpha}\right) T B^{\alpha} \\
& +\mathbf{g}_{, \alpha}(2 T+2 \lambda+\chi S) T B^{\alpha}+\mathbf{g}_{, \alpha} \lambda \chi S B^{\alpha} \\
= & (2 T+2 \lambda+\chi S) \mathbf{g}_{, \alpha} T B^{\alpha}+\lambda \chi \mathbf{g}_{, \alpha} S B^{\alpha} .
\end{aligned}
$$

Adding (7.4.10) to (7.4.13) plus their conjugates we obtain

$$
\left[H_{\Lambda} J\right]=(2 T+2 \lambda+\chi S) J
$$

as we wanted.

Finally, we need to check (3.13.2). For this we compute using the non-commutative Wick formula:

$$
\begin{aligned}
{\left[\Psi_{\alpha \Lambda} J\right] } & =i \chi \Psi_{\alpha}+i \mathbf{g}_{, \beta \alpha} T B^{\beta}+i \lambda \mathbf{g}_{, \alpha} \\
& =i \chi \Psi_{\alpha}+i(T+\lambda) \mathbf{g}_{, \alpha}, \\
{\left[J_{\Lambda} \Psi_{\alpha}\right] } & =-i(\chi+S) \Psi_{\alpha}-i \lambda \mathbf{g}_{, \alpha}, \\
{\left[S B_{\Lambda}^{\alpha} J\right] } & =-i \chi S B^{\alpha} \\
{\left[J_{\Lambda} S B^{\alpha}\right] } & =i(\chi+S) S B^{\alpha} .
\end{aligned}
$$

Here in the second line we used the fact that $M$ is Ricci-flat, therefore $\mathbf{g}_{, \alpha \bar{\beta}}=0$. Using this we can compute now

$$
\begin{aligned}
{\left[J_{\Lambda} S B^{\alpha} \Psi_{\alpha}\right]=} & i\left((\chi+S) S B^{\alpha}\right) \Psi_{\alpha} \\
& +i S B^{\alpha}(\chi+S) \Psi_{\alpha}+i S B^{\alpha} \lambda \mathbf{g}_{, \alpha}+\int_{0}^{\Lambda} i\left[(\chi+S) S B_{\Gamma}^{\alpha} \Psi_{\alpha}\right] d \Gamma \\
= & i T B^{\alpha} \Psi_{\alpha}+i S B^{\alpha} S \Psi_{\alpha}+i \lambda S B^{\alpha} \mathbf{g}_{, \alpha}+i n \int_{0}^{\Lambda}(\eta-\chi) \eta d \Gamma \\
= & i T B^{\alpha} \Psi_{\alpha}+i S B^{\alpha} S \Psi_{\alpha}+i \lambda S B^{\alpha} \mathbf{g}_{, \alpha}+i n \lambda \chi .
\end{aligned}
$$

Similarly we have

$$
\begin{aligned}
{\left[\mathbf{g}_{, \alpha} J\right] } & =-i S B^{\beta} \mathbf{g}_{, \alpha \beta} \\
& =-i S \mathbf{g}_{, \alpha}, \\
{\left[J_{\Lambda} \mathbf{g}_{, \alpha}\right] } & =-i S \mathbf{g}_{, \alpha}, \\
{\left[J_{\Lambda} T B^{\alpha}\right] } & =-i(\lambda+T) S B^{\alpha} .
\end{aligned}
$$

Hence using the Wick formula we obtain

$$
\begin{aligned}
{\left[J_{\Lambda} \mathbf{g}_{, \alpha} T B^{\alpha}\right] } & =-i\left(S \mathbf{g}_{, \alpha}\right) T B^{\alpha}-i \mathbf{g}_{, \alpha}(\lambda+T) S B^{\alpha} \\
& =-i \lambda \mathbf{g}_{, \alpha} S B^{\alpha}-i S\left(\mathbf{g}_{, \alpha} T B^{\alpha}\right) .
\end{aligned}
$$




\section{SUPERSYMmetRY OF THE CHIRAL DE RHAM COMPLEX}

Adding (7.4.18) and (7.4.16) plus their conjugates, we obtain

$$
\begin{aligned}
{\left[J_{\Lambda} J\right] } & =-T B^{i} \Psi_{i}-S B^{i} S \Psi_{i}-\lambda \chi 2 n+S\left(\mathbf{g}_{, \alpha} T B^{\alpha}\right)+S\left(\mathbf{g}_{, \bar{\alpha}} T B^{\bar{\alpha}}\right) \\
& =-T B^{i} \Psi_{i}-S B^{i} S \Psi_{i}+S T \mathbf{g}-2 n \lambda \chi .
\end{aligned}
$$

(iii) In order to prove the rest of the statement, we may choose holomorphic coordinates for one of the three complex structures. We suppose then that $J_{\omega}$ is given by (7.4.6). This forces the other two complex structures to be of the form

$$
\eta_{i}^{j}=\left(\begin{array}{cc}
0 & \eta_{\alpha}^{\bar{\beta}} \\
\eta_{\bar{\alpha}}^{\beta} & 0
\end{array}\right), \quad \gamma_{i}^{j}=\left(\begin{array}{cc}
0 & i \eta_{\alpha}^{\bar{\beta}} \\
-i \eta_{\bar{\alpha}}^{\beta} & 0
\end{array}\right)
$$

with $\eta_{\alpha}^{\bar{\beta}} \eta_{\bar{\beta}}^{\gamma}=-\delta_{\alpha}^{\gamma}$. It follows then that $J^{+}=\frac{1}{2}\left(J_{\eta}-i J_{\gamma}\right)$ is given by

$$
J^{+}=\eta_{\alpha}^{\bar{\beta}} S B^{\alpha} \Psi_{\bar{\beta}},
$$

where we note that the second term in (7.2.1) vanishes in this case since $M$ is Kähler. Therefore the only non-vanishing components of the Christoffel symbols are $\Gamma_{\beta \gamma}^{\alpha}$ and $\Gamma_{\bar{\beta} \bar{\gamma}}^{\bar{\alpha}}$. The theorem will be proved then if we show that ${ }^{2}$

$$
\left[J_{\omega \Lambda} J^{+}\right]=i(2 \chi+S) J^{+} .
$$

In order to do so we compute (we denote $J=J_{\omega}$ )

$$
\begin{aligned}
& {\left[\eta_{\alpha \Lambda}^{\bar{\beta}} J\right]=-i \eta_{\alpha, \gamma}^{\bar{\beta}} S B^{\gamma}+i \eta_{\alpha, \bar{\gamma}}^{\bar{\beta}} S B^{\bar{\gamma}},} \\
& {\left[J_{\Lambda} \eta_{\alpha}^{\bar{\beta}}\right]=-i \eta_{\alpha, \gamma}^{\bar{\beta}} S B^{\gamma}+i \eta_{\alpha, \bar{\gamma}}^{\bar{\beta}} S B^{\bar{\gamma}} .}
\end{aligned}
$$

It follows then from (7.4.15) and the Wick formula that

$$
\left[J_{\Lambda} \eta_{\alpha}^{\bar{\beta}} S B^{\alpha}\right]=-i \eta_{\alpha, \gamma}^{\bar{\beta}} S B^{\gamma} S B^{\alpha}+i \eta_{\alpha, \bar{\gamma}}^{\bar{\beta}} S B^{\bar{\gamma}} S B^{\alpha}+i \eta_{\alpha}^{\bar{\beta}}(\chi+S) S B^{\alpha} .
$$

Since the complex structure is parallel we have

$$
\eta_{\alpha, \gamma}^{\bar{\beta}}=\Gamma_{\gamma \alpha}^{\delta} \eta_{\delta}^{\bar{\beta}}=\eta_{\gamma, \alpha}^{\bar{\beta}} .
$$

Therefore the first term in (7.4.24) vanishes and we have

$$
\left[J_{\Lambda} \eta_{\alpha}^{\bar{\beta}} S B^{\alpha}\right]=i \eta_{\alpha, \bar{\gamma}}^{\bar{\beta}} S B^{\bar{\gamma}} S B^{\alpha}+i \eta_{\alpha}^{\bar{\beta}}(\chi+S) S B^{\alpha} .
$$

Now conjugating (7.4.15) we see that

$$
\left[J_{\Lambda} \Psi_{\bar{\beta}}\right]=i(\chi+S) \Psi_{\bar{\beta}}+i \lambda \mathbf{g}_{, \bar{\beta}} .
$$

Now using the non-commutative Wick formula we obtain

$$
\begin{aligned}
{\left[J_{\omega \Lambda} J^{+}\right]=} & i\left(\eta_{\alpha, \bar{\gamma}}^{\bar{\beta}} S B^{\bar{\gamma}} S B^{\alpha}\right) \Psi_{\bar{\beta}}+i\left(\eta_{\alpha}^{\bar{\beta}}(\chi+S) S B^{\alpha}\right) \Psi_{\bar{\beta}} \\
& -i\left(\eta_{\alpha}^{\bar{\beta}} S B^{\alpha}\right)(\chi+S) \Psi_{\bar{\beta}}-i\left(\eta_{\alpha}^{\bar{\beta}} S B^{\alpha}\right) \lambda \mathbf{g}_{, \bar{\beta}} \\
& +i \int_{0}^{\Lambda}\left[\eta_{\alpha, \bar{\gamma}}^{\bar{\beta}} S B^{\bar{\gamma}} S B_{\Lambda}^{\alpha} \Psi_{\bar{\beta}}\right] d \Gamma+i \int_{0}^{\Lambda}\left[\eta_{\alpha}^{\bar{\beta}}(\chi+S) S B_{\Lambda}^{\alpha} \Psi_{\bar{\beta}}\right] d \Gamma
\end{aligned}
$$

Let us compute the integral term first. Clearly the second integral vanishes since $\Psi_{\bar{\beta}}$ commutes with $S B^{\alpha}$. The first term on the other hand is given by

$$
-\int_{0}^{\Lambda} \eta \eta_{\alpha, \bar{\beta}}^{\bar{\beta}} S B^{\alpha} d \Gamma=-\lambda \eta_{\alpha, \bar{\beta}}^{\bar{\beta}} S B^{\alpha} .
$$

\footnotetext{
${ }^{2}$ Note that the commutation relations with $J^{-}=\frac{1}{2}\left(J_{\eta}+i J_{\gamma}\right)$ follow by conjugation.
} 
Re-grouping the terms in (7.4.28) we obtain

$$
\left[J_{\omega \Lambda} J^{+}\right]=i(2 \chi+S) J^{+}-i \lambda\left(\eta_{\alpha}^{\bar{\beta}} \mathbf{g}_{, \bar{\beta}} S B^{\alpha}+\eta_{\alpha, \bar{\beta}}^{\bar{\beta}} S B^{\alpha}\right) .
$$

Finally, using again the fact that $\eta$ is parallel, we see immediately that

$$
\eta_{\alpha, \bar{\beta}}^{\bar{\beta}}=-\Gamma_{\bar{\beta} \bar{\gamma}}^{\bar{\beta}} \eta_{\alpha}^{\bar{\gamma}}=-\mathbf{g}_{, \bar{\gamma}} \eta_{\alpha}^{\bar{\gamma}} \text {. }
$$

This proves (7.4.22) and completes the proof of Theorem 7.4.

Remark 7.5. (i) In the Calabi-Yau case, choosing holomorphic coordinates $x^{\alpha}$ and anti-holomorphic coordinates $x^{\bar{\alpha}}$, we see that $\mathbf{g}=\mathbf{g}_{0}+\overline{\mathbf{g}}_{0}$ where $\mathbf{g}_{0}$ is holomorphic. The superfield $H$ can be decomposed as a sum of two terms $H_{0}+\bar{H}_{0}$, where

$$
H_{0}=S B^{\alpha} S \Psi_{\alpha}+T B^{\alpha} \Psi_{\alpha}-T S \mathbf{g}_{0} .
$$

The superfield $J$ decomposes in a similar way as a 'holomorphic' part $J_{0}$ and an 'anti-holomorphic' part $\bar{J}_{0}$. These fields are invariant under holomorphic changes of coordinates, and hence we obtain two commuting $N=2$ superconformal structures.

We note that these fields are different from the ones considered in [MSV99]. In the case of the Virasoro field, the correction given by $T S \mathbf{g}_{0}$ appeared in [Wit05, p. 16]. When the metric is flat (i.e. $\mathbf{g}_{\mathbf{0}}=0$ ) we obtain the same topological structure as in [MSV99].

(ii) When the manifold $M$ is complex but not Calabi-Yau, the decomposition $H=H_{0}+\bar{H}_{0}$ is not invariant under holomorphic changes of coordinates. Therefore our $N=1$ structure pairs in a non-trivial way the 'holomorphic' and 'anti-holomorphic' parts of the chiral de Rham complex of $M$.

(iii) The fields $H$ and $J$ are defined for any almost complex manifold $M$ (though they generate $N=2$ only when $M$ is Calabi-Yau). In particular, the field $J$ allows us to construct a Dolbeault resolution of the holomorphic chiral de Rham complex in terms of the differentiable one (see also [Kap05]). We plan to return to this matter elsewhere.

\section{ACKNowledgements}

DB would like to thank the Aspen Center for Physics for its hospitality. RH would like to thank his advisor, Victor Kac. MS would like to thank Edward Frenkel and Vassily Gorbounov for valuable discussions, and RIMS for their hospitality. In June 2005, MS attended a conference at the Erwin Schrödinger Institute, where Fyodor Malikov explained that he had obtained quasiclassical limits of the $N=1$ and $N=2$ structures on CDR. MS is very grateful for this, as well as other valuable discussions.

\section{REFERENCES}

AGF81 L. Alvarez-Gaumé and D. Z. Freedman, Geometrical structure and ultraviolet finiteness in the supersymmetric $\sigma$-model, Comm. Math. Phys. 80 (1981), 443-451.

BGL04 S. Bellucci, A. Galajinsky and E. Latini, Making the hyperkähler structure of $n=2$ quantum string manifest, Phys. Rev. D (3) 2 (2004), 70-75.

Fre99 D. S. Freed, Five lectures on supersymmetry (American Mathematical Society, Providence, RI, 1999).

FBZ01 E. Frenkel and D. Ben-Zvi, Vertex algebras and algebraic curves, first edition (American Mathematical Society, Providence, RI, 2001).

FL07 E. Frenkel and A. Losev, Mirror symmetry in two steps: $A-I-B$, Comm. Math. Phys. 269 (2007), 39-86. 


\section{SUPERSYMMETRY OF THE CHIRAL DE RHAM COMPLEX}

GMS03 V. Gorbounov, F. Malikov and V. Schechtman, Gerbes of chiral differential operators III, in The orbit method in geometry and physics, Marseille, 2000, Progress in Mathematics, vol. 213 (Birkhäuser, Boston, MA, 2003), 73-100.

GMS04 V. Gorbounov, F. Malikov and V. Schechtman, Gerbes of chiral differential operators II. Vertex algebroids, Invent. Math. 155 (2004), 605-680.

HK07 R. Heluani and V. G. Kac, Supersymmetric vertex algebras, Comm. Math. Phys. 271 (2007), $103-178$.

HKLR87 N. Hitchin, A. Karlhede, U. Lindström and M. Roček, Hyperkähler metrics and supersymmetry, Comm. Math. Phys. 108 (1987), 535-589.

Joy00 D. Joyce, Compact manifolds with special holonomy, Oxford Mathematical Monographs (Oxford University Press, Oxford, 2000).

Kac96 V. G. Kac, Vertex algebras for beginners, University Lecture Series, vol. 10 (American Mathematical Society, Providence, RI, 1996).

KW04 V. G. Kac and M. Wakimoto, Quantum reduction and representation theory of superconformal algebras, Adv. Math. 185 (2004), 400-458.

KV04 M. Kapranov and E. Vasserot, Vertex algebras and the formal loop space, Publ. Math. Inst. Hautes Études Sci. 100 (2004), 209-269.

Kap05 A. Kapustin, Chiral de Rham complex and the half-twisted sigma-model, Preprint (2005), hep-th/0504074.

LL07 B. Lian and A. Linshaw, Chiral equivariant cohomology I, Adv. Math. 209 (2007), 99-161.

MSV99 F. Malikov, V. Shechtman and A. Vaintrob, Chiral de Rham complex, Comm. Math. Phys. 204 (1999), 439-473.

Tam99 H. Tamanoi, Elliptic genera and vertex operator super-algebras, Lecture Notes in Mathematics, vol. 1704 (Springer, Berlin, 1999).

Wit05 E. Witten, Two-dimensional models with (0,2) supersymmetry: perturbative aspects, Preprint (2005), hep-th/0504078.

Zho00 J. Zhou, Superconformal vertex algebras in differential geometry I, Preprint (2000), math.DG/0006201.

Zum79 B. Zumino, Supersymmetry and Kähler manifolds, Phys. Lett. 27B (1979), 203.

David Ben-Zvi benzvi@math.utexas.edu

Department of Mathematics, University of Texas, Austin, TX 78712, USA

Reimundo Heluani heluani@math.mit.edu

Department of Mathematics, MIT, Cambridge, MA 02139, USA

Matthew Szczesny szczesny@math.bu.edu

Department of Mathematics and Statistics, Boston University, Boston, MA 02215, USA 